\title{
Metastatic SW620 colon cancer cells are primed for death when detached and can be sensitized to anoikis by the BH3-mimetic ABT-737
}

\author{
A Maamer-Azzabi ${ }^{1}$, 0 Ndozangue-Touriguine ${ }^{2}$ and $\mathrm{J}$ Bréard $^{*, 1}$
}

Anoikis, a Bax-dependent apoptosis triggered by detachment from the extracellular matrix, is often inhibited in metastatic cancer cells. Using a couple of isogenic human colon cancer cell lines derived either from the primary tumor (SW480) or from a lymph node metastasis (SW620), we found that only SW480 cells were sensitive to anoikis. Bim upregulation but not Mcl-1 degradation was determined to be a critical factor of anoikis initiation in SW480 cells. ERK-mediated phosphorylation targets Bim for ubiquitination and proteasomal degradation. A MEK inhibitor (PD0325901) was able to increase Bim expression in SW620 cells and to sensitize these cells to anoikis. Thus, in both cell lines anoikis is under the control of proteins of the Bcl-2 family. Most interestingly, the BH3-mimetic ABT-737 was found not only to increase the level of apoptosis in suspended SW480 cells but also to sensitize SW620 cells to anoikis. Accordingly, both cell lines cultured in suspension were found to be primed for death, as determined by the detection of Bcl-2:Bim and Bcl-xL:Bim complexes. In contrast, adherent SW480 and SW620 cells were resistant to $A B T-737$. This indicates that, whether or not they undergo anoikis, colon cancer cells that have detached from the extracellular matrix might go through a transient state, where they are sensitive to BH3 mimetics. This would confer to compounds such as Navitoclax or ABT-199 a therapeutic window where they could have anti-metastatic potential.

Cell Death and Disease (2013) 4, e801; doi:10.1038/cddis.2013.328; published online 12 September 2013

Subject Category: Cancer

Anoikis is a particular apoptotic death due to loss of appropriate cell adhesion, ${ }^{1-3}$ and tumor cells that acquire metastatic potential have developed mechanisms to resist anoikis. ${ }^{4,5}$ In spite of its unique definition, anoikis is essentially an apoptotic process. In keeping with classical apoptosis, anoikis involves either the intrinsic pathway, due to the perturbation of mitochondrial homeostasis, or the extrinsic pathway triggered by cell surface death receptors. ${ }^{3,6}$

Proteins of the B-cell leukemia/lymphoma 2 (Bcl-2) family are key arbiters of the commitment to apoptosis at the mitochondrion. ${ }^{2,7}$ This family consist of both pro- and antiapoptotic members, which all share sequence homology in their Bcl-2 homology (BH) domains. The anti-apoptotic proteins, including $\mathrm{Bcl}-2, \mathrm{Bcl}-2$-related gene, long isoform (Bcl-xL), Bcl-w and myeloid cell leukemia 1 (Mcl-1) contain $\mathrm{BH}$ domains 1-4. They are generally found on the outer mitochondrial membrane, where they function to inhibit the pro-apoptotic $\mathrm{Bcl}-2$ proteins. The pro-apoptotic proteins are divided into the multi-domain effectors Bcl-2-acssociated $\mathrm{x}$ protein (Bax), Bak and Bok (containing $\mathrm{BH}$ domains 1-3), or $\mathrm{BH} 3-o n l y$ proteins, which contain only the $\mathrm{BH} 3$ domain. The multi-domain pro-apoptotic proteins Bax and Bak promote mitochondrial outer membrane permeabilization. ${ }^{8}$
The BH3-only proteins, such as $\mathrm{Bcl}-2$ interacting domain death agonist (Bid), Bim and PUMA act as either direct activators of pro-apoptotic Bax and Bax, or as repressors of anti-apoptotic proteins. ${ }^{9,10}$ They have been named activators. The other class of BH3-only proteins such as Bad, termed sensitizers, triggers apoptosis by binding to anti-apoptotic proteins and displacing the activator $\mathrm{BH}$-only proteins.

ABT-737 is a rationally designed small molecule that binds with high affinity to $\mathrm{Bcl}-2$ and $\mathrm{Bcl}-\mathrm{xL}$ but not $\mathrm{Mcl}-1$, and antagonizes their anti-apoptotic function. ${ }^{11,12}$ ABT-737 has been shown to reverse acquired paclitaxel resistance in breast cancer cell lines. ${ }^{13}$ Combined with rapamycin, ABT-737 enhances the radio sensitivity of non-small cell lung tumors. ${ }^{14}$ The combination of ABT-737 and a MEK inhibitor leads to tumor regression in different mouse tumor models of KRAS mutant cells. ${ }^{15}$ In human myeloid leukemia cell lines and xenograft models, ABT-737 synergizes with inhibitors of the PI3K/AKT/mTOR pathway. ${ }^{16}$ Navitoclax (ABT-263), an orally available variant of ABT-737, is currently being evaluated in phase 2 clinical trials. Despite promising preliminary data, thrombocytopenia limits the ability to raise drug concentrations. Since then, ABT-199, a selective Bcl-2 inhibitor with no activity on $\mathrm{BCl}-\mathrm{xL}$, has been shown to achieve anti-tumor activity as a single agent in chronic lymphocytic

\footnotetext{
${ }^{1}$ Inserm U1004, Hôpital Paul Brousse, 12 Avenue Paul Vaillant-Couturier, Villejuif 94800, France and '2'OREAL - River Plaza, 25-29 quai Aulagnier, 92600 Asnières-sur-Seine, France

*Corresponding author: Dr J Bréard, Inserm U1004, Hôpital Paul Brousse, 12 Avenue Paul Vaillant-Couturier, Villejuif 94800, France. Tel: + 33145595275 ; Fax: + 3314726 0319; E-mail: jacqueline.breard@u-psud.fr

Keywords: anoikis; colon cancer; Bcl-2 family; metastasis; ABT-737

Abbreviations: Bcl-2, B-cell leukemia/lymphoma 2; Bax, Bcl-2-acssociated x protein; Bcl-xL, Bcl-2-related gene, long isoform; Mcl-1, myeloid cell leukemia 1; Bid, Bcl-2 interacting domain death agonist; Bim, B-cell lymphoma 2 interacting mediator of cell death; siRNA, short interfering RNA; EMT, epithelial-mesenchymal transition; FADD, Fas-associated death domain

Received 05.3.13; revised 30.7.13; accepted 01.8.13; Edited by H-U Simon
} 

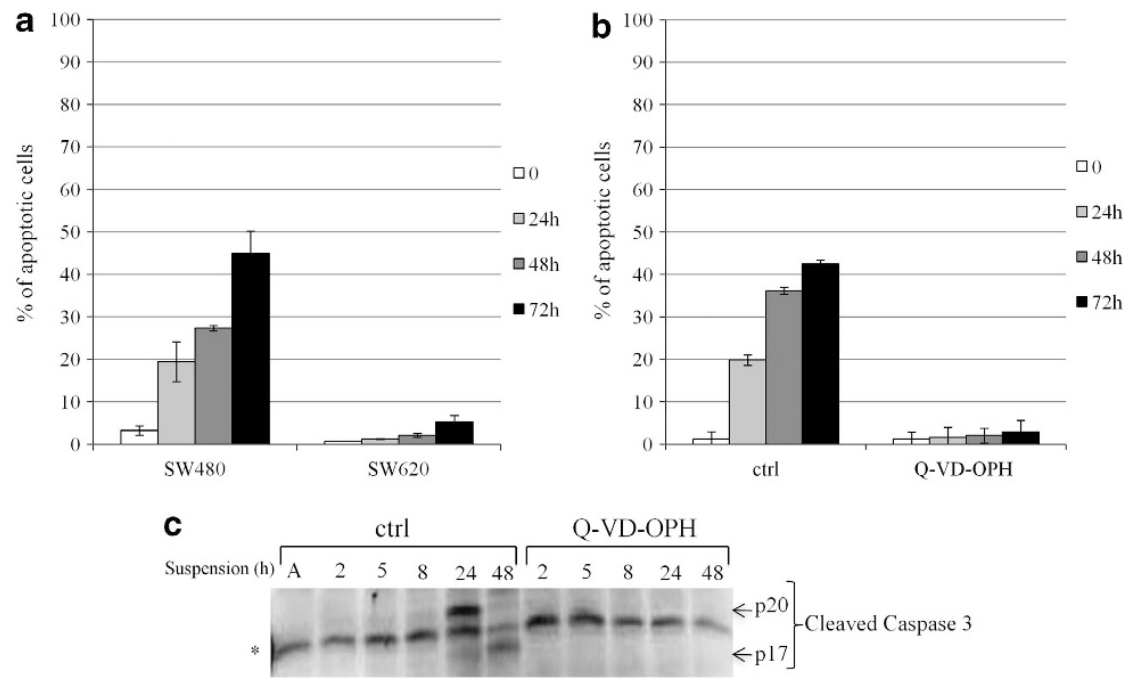

Figure 1 Anoikis sensitivity in SW480 and SW620 cells. (a) Time course of apoptotic cell death in suspended SW480 and SW620 cell populations. (b) Time course of apoptosis in suspended SW480 cells cultured without (ctrl) or with the pan-caspase inhibitor Q-VD-OPH (10 $\mu \mathrm{M})$. (c) Kinetics of caspase-3 activation in adherent (A) or suspended SW480 cells cultured without (ctrl) or with Q-VD-OPH $(10 \mu \mathrm{M})$ detected by means of western blot with an anti-cleaved-caspase-3 antibody (p20 and p17 fragments). The asterisk denotes a nonspecific band. For all graphs in all figures, error bars indicate S.D. of at least three independent experiments

leukemia while sparing platelets. ${ }^{17}$ The BH3-mimetic ABT-737 and related compounds act as single agents to induce apoptosis only in cancer cells that are dependent on Bcl-2 or Bcl-xL for survival. ${ }^{18,19}$ This dependence appears to correlate with sequestration of activator $\mathrm{BH} 3$-only proteins by the anti-apoptotic proteins and such cells have been defined as 'primed for death'. BH3 mimetics, by displacing the activator $\mathrm{BH} 3$-only proteins from $\mathrm{Bcl}-2$ or $\mathrm{BCl}-\mathrm{xL}$, allow their interaction with and activation of Bax or Bak. In cells where $\mathrm{Bcl}-2$ and $\mathrm{Bcl}-\mathrm{xL}$ are not primed, exposure to chemotherapeutic agents has often been shown to induce the expression of activator BH3-only proteins leading to a synergy between these agents and $\mathrm{BH} 3$ mimetics.

It has been reported that when cultured in suspension, some anoikis-sensitive cells induce the expression of the BH3-only proteins B-cell lymphoma 2 interacting mediator of cell death $(\mathrm{Bim})^{20}$ or Bmf. ${ }^{21}$ We therefore reasoned that a parallel could be drawn with the effects of chemotherapeutics and investigated whether, when they are detached, the cell sensitivity to $\mathrm{BH} 3$ mimetics is modified. To this end, we used a pair of isogenic colon carcinoma cell lines: SW480 derived from a primary Duke's stage B colon carcinoma, and SW620 derived from a mesenteric lymph node metastasis in the same patient. ${ }^{22}$ SW480 cells are sensitive to anoikis, whereas SW620 cells are resistant. Bim was found to be upregulated in suspension in SW480 cells and to mediate, at least in part, their sensitivity to anoikis. Most interestingly, both cell lines died in the presence of ABT-737 when cultured in suspension, whereas they were resistant to this compound under adherent conditions. Thus, detached colon cancer cells are primed for death, whether or not they are sensitive to anoikis, and compounds such as ABT-263 or ABT-199 might therefore have anti-metastatic properties.

\section{Results}

SW480 cells are sensitive to anoikis unlike SW620 cells. The time course level of anoikis was compared in
SW480 and SW620 cells following culture in suspension. As shown in Figure 1a, the percentage of SW480 apoptotic cells increased with time. In contrast, apoptosis in SW620 cells was barely detectable. In order to check whether SW480 cell death was due to classical apoptosis, cells were cultured in the presence or absence of the pan-caspase inhibitor $\mathrm{Q}-\mathrm{VD}-\mathrm{OPH}$. The results shown in Figure $1 \mathrm{~b}$ indicate that this inhibitor fully protected SW480 cells against detachmentinduced cell death. We also monitored caspase-3 activation in suspended SW480 cells by the detection of the p20 (intermediate) and p17 (mature) fragments of the active caspase through western blot analysis. The p20 fragment and some p17 fragment were detected at $24 \mathrm{~h}$ (Figure 1C) and by $48 \mathrm{~h}$ caspase-3 was fully mature. The presence of Q-VD-OPH totally abrogated caspase-3 activation (Figure 1c).

Anoikis has been ascribed to the intrinsic ${ }^{23}$ or to the extrinsic $^{24,25}$ apoptotic pathway. We therefore studied in kinetics the cleavage and thus the activation of caspase-8 and caspase-9. As can be seen in Figure 2a we detected a faint cleavage band for both caspases as soon as $5 \mathrm{~h}$ of culture in suspension. In order to further test which initiator caspase was at the apex of the apoptotic signaling, we used two clones of SW480 cells stably transfected with an expression plasmid encoding for $\mathrm{Bcl}-2{ }^{26}$ Both clones were resistant to anoikis (Figure $2 \mathrm{~b}$ ) and, most importantly, activation of all caspases was abrogated in these clones (Figure 2c) indicating that the activation of caspase-8 shown in Figure $2 \mathrm{a}$ occurs downstream of mitochondrial events. To further document that the extrinsic pathway was not involved, SW480 cells were stably transfected with an expression plasmid encoding a dominantnegative form of Fas-associated death domain (FADD) (FADD-dn) and three clones were obtained (Figure 2d). All the clones showed resistance to TRAIL-induced apoptosis, as illustrated in Figure $2 \mathrm{e}$ for clone 4. However, all clones were sensitive to anoikis, as illustrated in Figure $2 f$ for clone 4, ruling out the extrinsic pathway. 
a
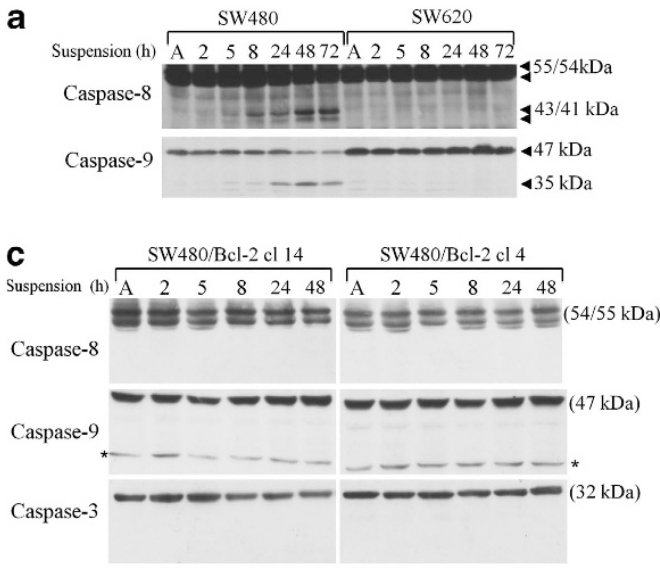

e

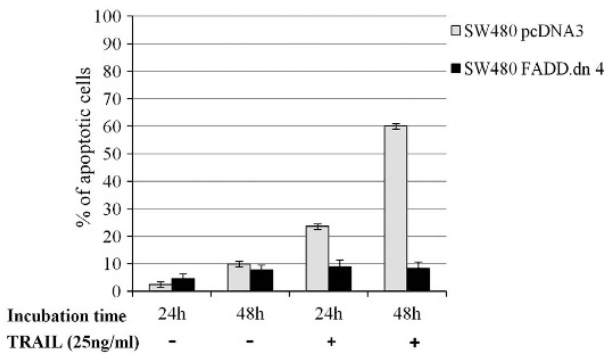

b

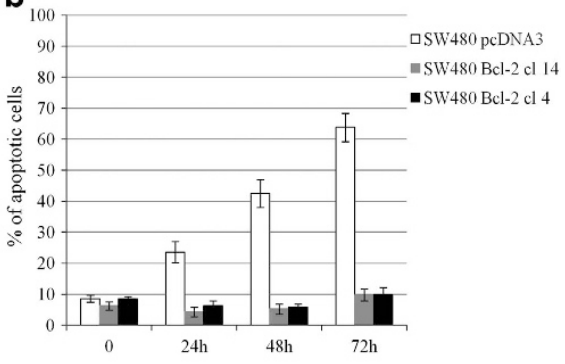

d

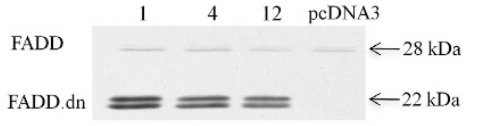

f

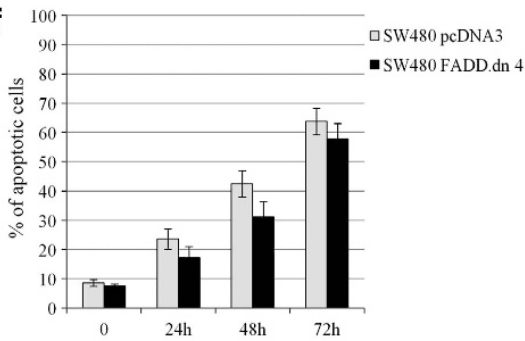

Figure 2 Anoikis in SW480 cells is initiated at the mitochondria. (a)Kinetics of initiator caspase activation in SW480 and SW620 cell lines cultured in suspension. Fulllength $(55 / 54 \mathrm{kD})$ or cleaved $(43 / 41 \mathrm{kD})$ caspase-8 and full-length $(47 \mathrm{kD})$ or cleaved $(35 \mathrm{kD})$ caspase-9 are detected. (b) Percentage of anoikis in empty vector or Bcl-2transfected SW480 clones. (c) Lack of detection of caspase activation in Bcl-2-transfected clones during the culture in suspension. Nonspecific band is represented by * (d) Cell extracts of SW480 clones transfected with FADD.dn (clones 1,4 and 12) or with empty vector (pcDNA3) were blotted as described in Materials and Methods with an anti-FADD antibody (e, f) SW480 cells transfected with empty vector (SW480 pcDNA3) or expressing FADD.dn (clone 4) were either cultured in the presence of recombinant TRAIL $(25 \mathrm{ng} / \mathrm{ml})(\mathbf{e})$ or cultured in suspension (f), and apoptosis was quantified at various times

SW620 cells' resistance to anoikis is not due to epithelialmesenchymal transition. Epithelial-mesenchymal transition (EMT) is not only a key event for epithelial cells to acquire a motile phenotype but it also allows cancer cells to avoid anoikis. ${ }^{27}$ During the EMT process, E-cadherin expression is lost, whereas $\mathrm{N}$-cadherin expression is upregulated, a process referred to as the 'cadherin switch'. ${ }^{28}$ Both the loss of E-cadherin ${ }^{29}$ and the acquisition of $\mathrm{N}$-cadherin ${ }^{30}$ expression appear important for the generation of survival signals. SW480 cells display an epithelial morphology, whereas SW620 cells have a rounded or fibroblast-like morphology reminiscent of cells having undergone EMT. ${ }^{31}$ The expression of various epithelial and mesenchymal markers was therefore studied in both cell lines. As shown in Figure 3, the expression of E-cadherin was slightly diminished in SW620 cells compared with SW480 cells but cytokeratin 18, another epithelial marker was greatly increased in SW620 cells. Concerning mesenchymal markers, vimentin was present in both cell lines but neither $\mathrm{N}$-cadherin nor fibronectin was expressed, whereas both were found in murine myeloblasts. Thus, SW620 cells do not seem to be of mesenchymal nature.

The BH3-only protein Bim has a role in SW480 cell death due to anoikis. Given that mitochondrial apoptosis is governed by members of the Bcl-2 family, the kinetic of expression of several of these proteins was studied in both SW480 and SW620 cell lines following their culture in

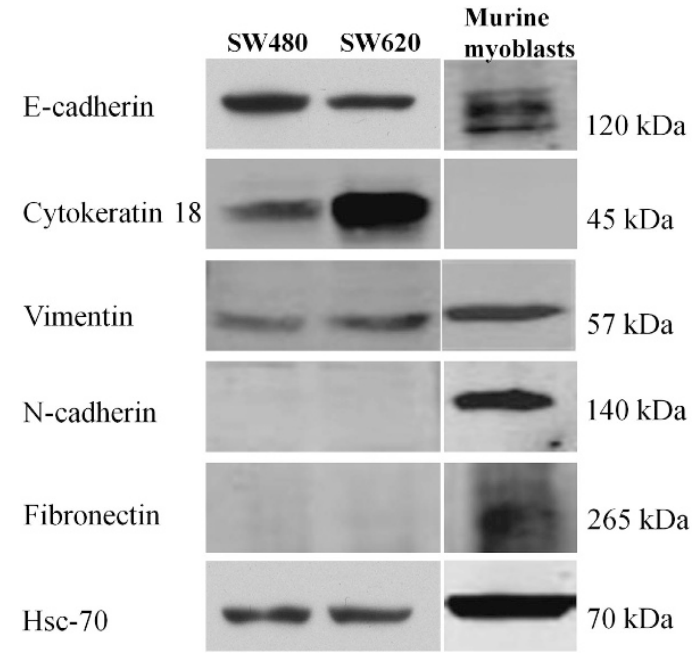

Figure 3 Expression of various epithelial or mesenchymal markers in SW480 and SW620 cells. Cell extracts were blotted with various antibodies recognizing epithelial (E-cadherin, Cytokeratin 18) or mesenchymal (vimentin, N-cadherin, fibronectin) markers. Murine myeloblasts were used as a positive control for mesenchymal cells

suspension. As shown in Figure 4a, the anti-apoptotic Bcl-2 and $\mathrm{BCl}-\mathrm{xL}$ were expressed at comparable levels in both cell lines and their expression did not vary during the culture in 

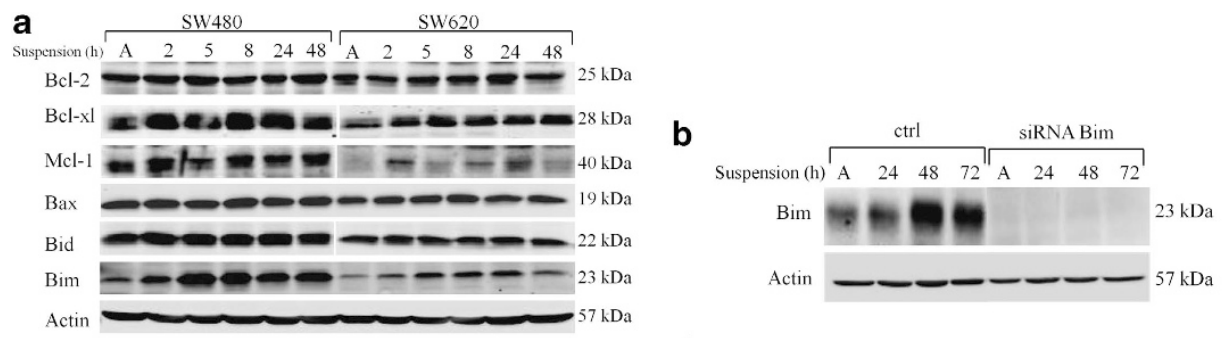

C

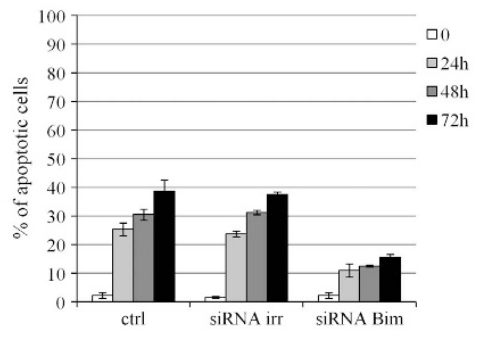

e

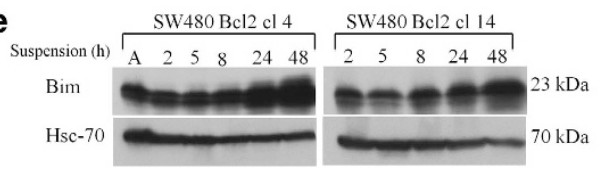

\section{。}

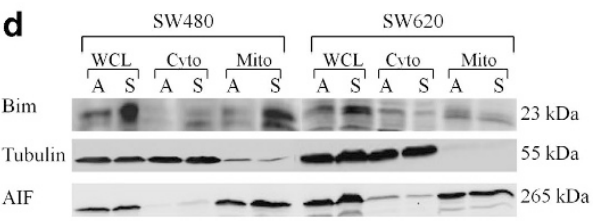

f

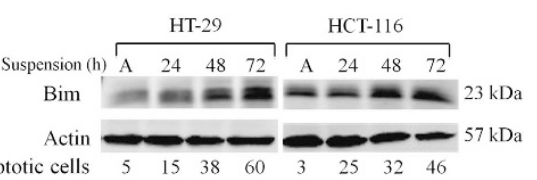

Figure 4 Study of the expression of Bcl-2 protein family in SW480 and SW620 cells cultured in suspension. (a) Time course analysis of the expression of several members of the Bcl-2 protein family analyzed by means of western blot in adherent (A) or suspended SW480 and SW620 cells. (b) Bim knockout efficiency in adherent (A) or suspended SW480 cells treated (siRNA Bim) or not (ctrl) with a specific Bim siRNA monitored by means of western blot using a specific anti-Bim antibody. (c) Percentage of apoptosis in SW480 cell populations cultured in suspension either untransfected (ctrl) or following transfection with a specific Bim siRNA (siRNA Bim) or siRNA to an irrelevant target (siRNA irr). (d) Analysis of Bim expression in whole cell lysates and in the cytosolic (cyto) or membrane/organellar (mito)-enriched fractions in adherent cells (A) or after $24 \mathrm{~h}$ of suspension (S). (e) Time course analysis of the expression of Bim in SW480 overexpressing Bcl-2 (SW480 Bcl-2 4, SW480 Bcl-2 14) either adherent (A) or cultured in suspension. (f) Bim expression in malignant colorectal HT-29 and HT-116 cell lines cultured in suspension

suspension. Mcl-1 expression did not change during the culture in SW480 cells in contrast with a recent report that found a substantial reduction of $\mathrm{Mcl}-1$ expression in $\mathrm{NIH} 3 \mathrm{~T} 3$ cells during anoikis. ${ }^{32}$ Overall, in our study, the anoikis-resistant SW620 cells expressed less Mcl-1 than SW480 cells and, for unknown reasons, its level of expression appeared sometimes to fluctuate during the culture. This result might be due to the very short Mcl-1 half-life $(40 \mathrm{~min}){ }^{33}$ Among the pro-apoptotic proteins, Bax and Bid expression levels were stable in suspended cells. In contrast, the expression of the BH3-only protein Bim greatly increased during the culture in suspension of SW480 cells and within $5 \mathrm{~h}$ after cell detachment. Of note is the fact that mostly the Bim-EL variant ${ }^{34}$ was detected in these cells and is referred to as Bim throughout the study. Bim expression was increased in SW620 but to a lesser degree.

We abrogated Bim expression in SW480 cells with a specific small-interfering RNA. As shown in Figure 4b, Bim expression was fully inhibited by this treatment throughout the culture in suspension. Lack of Bim was found to greatly but not totally protect SW480 cells from anoikis (Figure 4c). Therefore, we can conclude that Bim is involved in the sensitivity of SW480 cells to anoikis. Bmf has been implicated in the death due to anoikis of several cell types. ${ }^{21,35,36}$ However, we could not detect Bmf by western blot analysis in any of the two cell lines (not shown).

In healthy cells, Bim-EL can be sequestered to the microtubule-associated dynein motor complex through its binding to LC8. ${ }^{37}$ Certain apoptotic stimuli disrupt the interaction between LC8 and the dynein motor complex, thereby freeing Bim and thus enabling its translocation to the mitochondria. We therefore sought to determine whether, in addition to Bim level in suspended cells, there might also be differences in its localization in SW480 cells compared with SW620 cells. Microtubules depolymerize within a few minutes at room temperature and at $4{ }^{\circ} \mathrm{C}$ and therefore cannot be isolated by centrifugation. We utilized another approach with the use of the 'cell fractionation kit' as described in Material and Methods. The cell extracts that we obtained were clean, as monomeric tubulin was found exclusively in the cytosolic protein-enriched fraction, whereas AIF, a mitochondrial protein, was detected exclusively in the membrane and organellar protein-enriched fraction (Figure 4d). Interestingly, Bim in adherent SW480 cells was found mainly in the membrane and organellar protein-enriched fraction, and upregulated Bim in suspended cells was detected in this same fraction. Therefore, the increased amount of Bim that we find in detached SW480 cells is largely localized in the mitochondria. In contrast, Bim was found to distribute roughly equally in the cytosolic and membrane protein-enriched fractions in adherent and suspended SW620 cells. These results suggest that there might be a less efficient translocation of Bim to the mitochondria in SW620 cells.

We then tested the two Bcl-2 expressing SW480 clones that we found to be resistant to anoikis. Both clones upregulated Bim when cultured in suspension (Figure 4e) in the same order of magnitude compared with the parental cells (Figure 3a). 

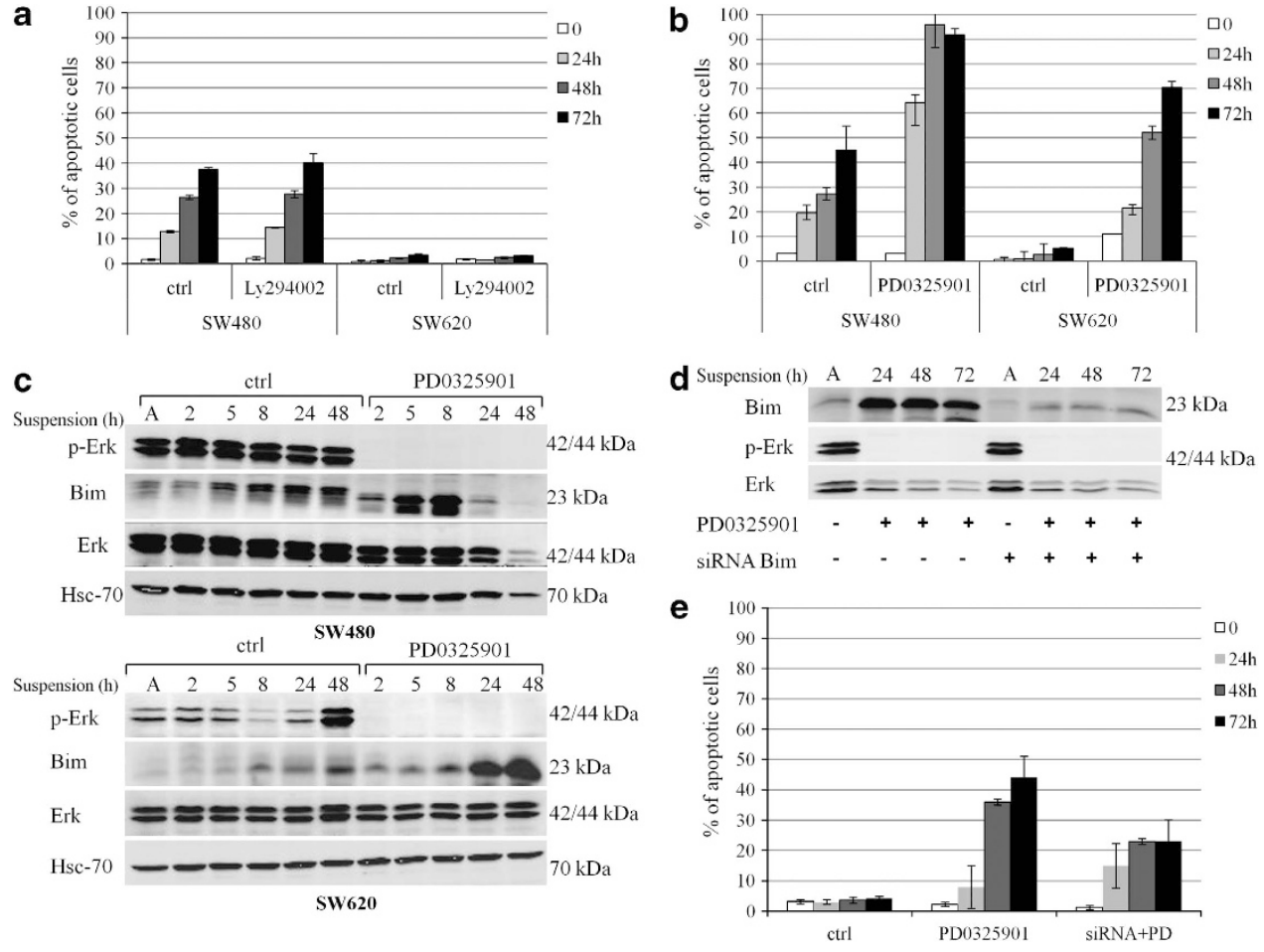

Figure 5 Regulation of Bim protein expression. (a) Percentage of apoptosis in suspended SW480 and SW620 cells cultured in the presence (Ly294002) or absence (ctrl) of the PI3K inhibitor Ly294002 $(10 \mu \mathrm{M})$. (b) Percentage of apoptosis in suspended SW480 and SW620 cells cultured in the presence (PD0325901) or absence (ctrl) of the MEK inhibitor PD0325901 (10 $\mu \mathrm{M})$. (c) Western blot analysis of the expression of p-ERK, Bim and total ERK in adherent (A) or suspended SW480 (upper panel) and SW620 (lower panel) cells cultured in the presence (PD0325901) or absence (ctrl) of PD0325901 (10 $\mu \mathrm{M}$ ). Anti-Hsc-70 was used as a loading control. (d) Western blot analysis of Bim expression in PD0325901-treated SW620 cells incubated with or without Bim siRNA. (e) Time course of anoikis level in PD0325901-treated SW620 cells incubated or not with Bim siRNA

Therefore, the sensitivity of SW480 cells to anoikis is not solely dictated by the level of expression of Bim but by the ratio of Bim to Bcl-2.

Finally, we used two other malignant colorectal cell lines to check whether Bim upregulation in suspended cells was a general feature. As shown in Figure $4 \mathrm{f} \mathrm{Bim} \mathrm{expression} \mathrm{was}$ increased in both HT-29 and HCT-116 cells during the culture in suspension, and this was accompanied by a significant cell death due to anoikis.

ERK inhibition in SW620 cells restores Bim expression in detached cells and their sensitivity to anoikis. The expression of Bim is controlled by both transcriptional and posttranscriptional mechanisms. ${ }^{38}$ The forkhead-like transcription factor $\mathrm{FOXO}$ a (forkhead box O3a) is a key transcriptional regulator of Bim and its activity is suppressed through its phosphorylation by Akt. As shown in Figure 5a, blocking of the PI3K/Akt pathway did not sensitize SW620 cells to anoikis. Moreover, western blot analysis using an anti-phospho-Akt antibody, revealed very little amount of activated Akt in SW620 cells (not shown).

On the other hand, ERK-mediated phosphorylation targets Bim for ubiquitylation and proteasomal degradation. An MEK inhibitor (PD0325901) was very efficient in sensitizing SW620 cells to anoikis while also increasing the level of cell death in SW480 cells cultured in suspension (Figure 5b). In SW620 cells (Figure 5c), ERK basal activity, as evaluated by western blot analysis of phosphorylated ERK ( $p$-ERK), was low and increased at the end of the culture, a finding that we repeatedly observed. The MEK inhibitor was very efficient in abrogating ERK activity and this was accompanied by an upregulation of Bim expression starting at $24 \mathrm{~h}$ correlating with anoikis observed at these time points.

We silenced the expression of Bim by RNA interference in suspended SW620 cells cultured in the presence of PD0325901 (Figure 5d). As shown in Figure 5e, extinction of Bim expression decreased the percentage of apoptosis by about half at $72 \mathrm{~h}$ of PD0325901 treatment indicating that Bim is involved in the sensitization to anoikis by this compound. These data also indicate that additional mechanisms are responsible for the remaining cell death. Surprisingly, ERK activity was high and sustained in control SW480 cells throughout the culture in suspension (Figure $5 \mathrm{c}$ ) but did not prevent the upregulation of Bim expression in control SW480 cells starting at $5 \mathrm{~h}$. MEK inhibition induced a stronger augmentation of Bim expression (Figure $5 \mathrm{c}$ ). In these conditions, Bim electrophoretic mobility was increased due to the fact that Bim is dephosphorylated. Bim was not detectable at late time points, an observation that we believe to be linked to the very high death rate, which affects the quality of the cell extracts. Likewise, total Erk and Hsc-70, the loading control, were diminished at these late time points.

ABT-737 sensitizes both SW480 and SW620 cells to anoikis. The upregulation of Bim in SW480 cells cultured in suspension led us to test whether, as has been shown for 

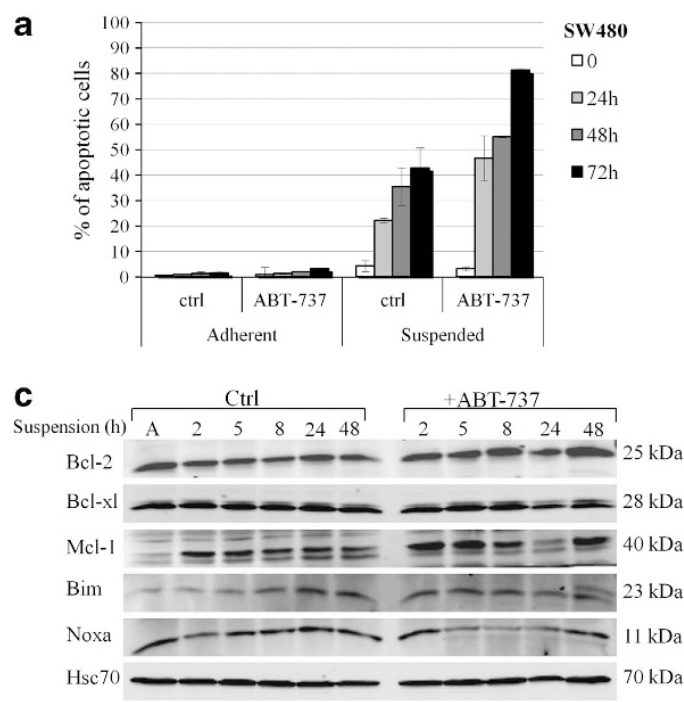

e

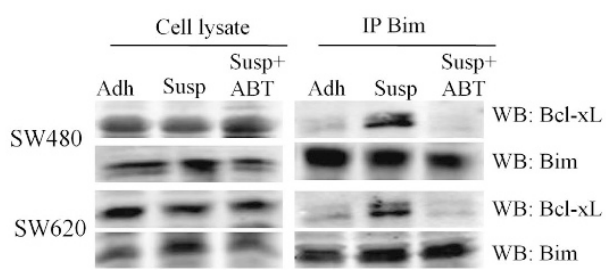

b

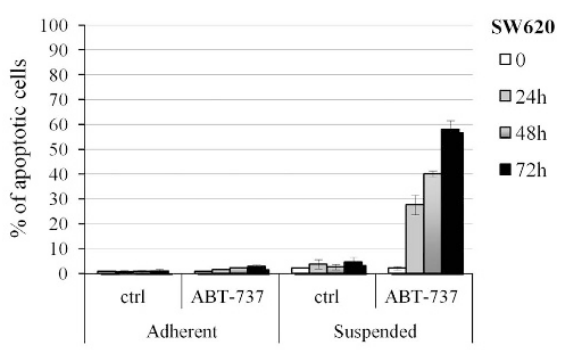

d $\quad \frac{\text { Cell lysate }}{\text { Susp }^{+}} \stackrel{\text { IP Bim }}{\text { Susp }}+$

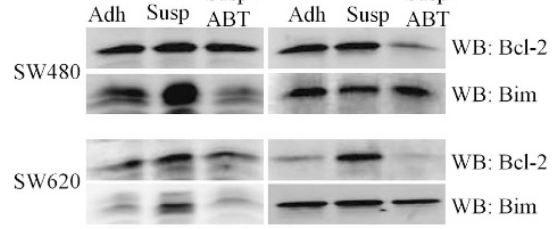

f

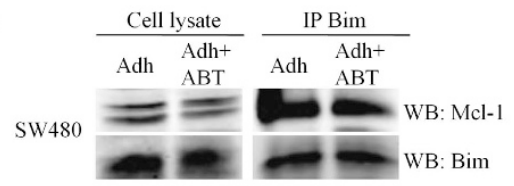

Figure 6 Analysis of SW480 and SW620 cell sensitivity to the BH3-mimetic ABT-737. (a, b) Percentage of apoptosis in adherent or suspended SW480 (a) or SW620 (b) cells cultured in the presence (ABT-737) or absence (ctrl) of ABT-737 $(1 \mu \mathrm{M})$. (c) Analysis of the Bcl-2 family member expression in SW620 cells during the culture in suspension in the presence or absense of ABT-737. (d) SW480 (upper panels) and SW620 (lower panels) cells either adherent (Adh) or suspended for $24 \mathrm{~h}$ in the presence (Susp + ABT) or absence (Susp) of ABT-737 $(1 \mu \mathrm{M})$ were lysed in CHAPS buffer. Cleared lysates were either analyzed directly (cell lysate) or following immunoprecipitation with anti-Bim antibody (IP Bim) and immunoblotted (WB) for Bcl-2 and Bim as described in Materials and Methods. (e) Cells were treated as in $\mathbf{d}$ and analyzed for Bcl-xL co-immunoprecipitation. (f) Adherent SW480 cells cultured in the presence or absence of ABT-737 for $24 \mathrm{~h}$ were lysed as in $\mathbf{d}$ and analyzed for Mcl-1 co-immunoprecipitation

numerous chemotherapeutic drugs used together with Navitoclax, ${ }^{39}$ a synergy could be found between the suspended state and ABT-737. As shown in Figure 6a, adherent SW480 cells were totally resistant to ABT-737 $(1 \mu \mathrm{M})$. However, in suspended cells, the presence of ABT-737 was found to greatly increase the level of anoikis. The same type of experiments were performed in SW620 cells (Figure 6b). Again, adherent cells were resistant to ABT-737. Surprisingly, SW620 cells cultured in suspension, despite their resistance to anoikis, died in significant numbers in the presence of ABT-737. We therefore checked whether the presence of ABT-737 could have modified the expression of some Bcl-2 family members in these cells. As shown in Figure 6c, this was not the case. These observations are particularly important concerning Noxa, as this Mcl-1 inhibitor has been reported to be induced by ABT-737 treatment in a glioma cell line. ${ }^{40}$

Cells sensitive to ABT-737 are supposed to be primed for death with $\mathrm{Bcl}-2$ and/or $\mathrm{Bcl}-\mathrm{xL}$ complexed to activator $\mathrm{BH} 3-$ only proteins. ${ }^{41}$ Immunoblots of $\mathrm{Bim}$ and $\mathrm{Bcl}-2$ or $\mathrm{Bcl}-\mathrm{xL}$ were therefore performed in whole cell lysates and in anti-Bim immunoprecipitates in SW480 and SW620 cell lines. As shown in Figure 6d, Bcl-2:Bim complexes were detected in both cell lines cultured in suspension. The formation of these complexes was abrogated in the presence of ABT-737, indicating that this compound displaced Bim from $\mathrm{Bcl}-2$ (Figure 6d). Most importantly, Bcl-xL:Bim complexes were also detected in suspended SW480 and SW620 cells and displaced by ABT-737 (Figure 6e). Bcl-2:Bim complexes were also found in adherent SW480 cells (Figure 6d) despite their lack of sensitivity to ABT-737 in these culture conditions. It is possible that, in the absence of Bim upregulation, the amount of such complexes is too low to offset the anti-apoptotic function of Mcl- $1^{42}$, which is highly expressed in SW480 cells (Figure 3a). We performed immunoprecipitations of Bim in adherent SW480 cells treated or not with ABT-737, which revealed the presence of Bim:Mcl-1 complexes in both conditions (Figure 6f). Therefore, we believe that, as Bim level is low in adherent SW480 cells, Mcl-1 is able to capture the small amount of protein displaced from $\mathrm{Bcl}-2 / \mathrm{Bcl}-\mathrm{xL}$ by ABT-737.

\section{Discussion}

Anoikis is by essence an anti-metastatic process and resistance to this form of cell death correlates with cancer aggressiveness. ${ }^{4}$

In this study, we used the SW480 and SW620 cell lines that have been validated as a model of colon cancer progression from primary tumor (SW480) to metastatic cells $(\mathrm{SW620})^{31}$, and show that during this progression the tumor cells have acquired an anoikis-resistant phenotype. EMT has been shown to be an important phenomenon for the acquisition of anoikis resistance ${ }^{33}$; however, we did not find any evidence 
that this process had occurred in SW620 cells despite their suggestive fibroblast-like morphology. It remains possible that these metastatic cells have undergone transient EMT followed by mesenchymal-epithelial transition once they have colonized their secondary site, as has been suggested in invasive colon cancer. ${ }^{44}$

Among the pro-apoptotic proteins tested, Bim was the only one to be highly upregulated in suspended SW480 cells. Importantly, silencing of Bim expression confirmed that this $\mathrm{BH} 3-$ only protein was involved in SW480 cell death by anoikis. However, the level of apoptosis went from $38 \%$ in control cells to $15 \%$ in cells treated with the Bim short interfering RNA (siRNA) indicating that another pathway might be implicated in this remaining cell death. We determined that an implication of Bmf was unlikely.

Most interestingly, when the effect of ABT-737 on suspended cells was evaluated, this compound was found to enhance anoikis not only in SW480 cells, which upregulate Bim in suspension, but also in SW620 cells, which only slightly do so. Several studies have been performed to determine ABT-737 mode of action. One of these studies, in acute myeloid leukemia, indicates that ABT-737 acts by inducing a dissociation of $\mathrm{Bcl}-2$ :Bax complexes and induces apoptosis in a Bak-dependent but Bim-independent manner. ${ }^{45}$ However, we have been unable to detect such complexes in either cell line in adhesion or in suspension (not shown). On another hand, in both cell lines cultured in suspension, Bcl-2:Bim and $\mathrm{Bcl}-\mathrm{xL}$ :Bim complexes were immunoprecipitated and these complexes were disrupted by ABT-737. Our data are therefore more in accordance with results obtained in chronic lymphocytic leukemia, where liberation of Bim from Bcl-2 by ABT-737 leads to Bax activation. ${ }^{19}$ Thus, Bim occupation of $\mathrm{Bcl}-2$ and $\mathrm{Bcl}-\mathrm{xL}$ in suspended cells primed SW480 and SW620 cells for death and ABT-737 acted by freeing Bim. Bcl-2:Bim or $\mathrm{Bcl}-\mathrm{xL}$ :Bim complexes were hardly detected in adherent SW620 cells, likely explaining why they were resistant to ABT-737. In contrast, adherent SW480 cells displayed $\mathrm{Bcl}-2$ :Bim complexes, whereas these cells were not sensitive to ABT-737. Mcl-1 has been shown to be a key determinant of the resistance to ABT-737 45,46 , and SW480 cells express high levels of $\mathrm{Mcl}-1$. Therefore, even if $\mathrm{Bcl}-2$ :Bim complexes were disrupted by ABT-737 in these adherent cells, the displaced Bim could be bound by Mcl-1, maintaining survival. Indeed, we could detect Mcl-1:Bim complexes in adherent SW480 cells treated or not with ABT-737. Upregulation of Bim in the suspended SW480 cells should allow for more Bcl-2:Bim complexes to be formed. In this case, the amount of Bim freed by ABT-737 would subvert the buffering capacity of $\mathrm{Mcl}-1$, thus rendering the cells sensitive to the $\mathrm{BH} 3$-mimetic.

Bim steady-state level is regulated through its ERK1/2dependent phosphorylation, which targets it for degradation by the proteasome. ${ }^{47}$ Inhibition of MEK was found to upregulate Bim expression in both cell lines and to sensitize SW620 cells to anoikis. Thus, SW620 cells in suspension are sensitive to Bim-induced apoptosis provided that this $\mathrm{BH}$ only protein is present in sufficient amounts. However, it appears unlikely that the ERK pathway is solely responsible for the lack of Bim upregulation in suspended SW620 cells, as we did not observe a strict negative correlation between the p-ERK level and Bim expression. Indeed, ERK activity is low in these cells and increased only at later time points. Given our results that the PI3K-Akt pathway is also not involved, it is likely that another pathway, which remains to be identified, is also at play in the regulation of Bim expression.

$\mathrm{Mcl}-1$ level, high and stable during the culture in suspension of SW480 cells was lower and sometimes fluctuating in SW620 cells. It is interesting to note that Mcl-1 degradation has been shown to be necessary for anoikis induction in some models. This is clearly not the case in SW480 cells. The short half-life of $\mathrm{Mcl}-1$ has been ascribed to its phosphorylation by GSK-3 followed by its ubiquitination and degradation by the proteasome. ${ }^{48}$ GSK-3 is inhibited by the PI3K-Akt pathway. We found very little Akt activity in SW620 cells, and GSK-3 might therefore be responsible for the reduced amount of Mcl-1 expressed by SW620 cells. In any case, low level of Mcl-1 would be expected to sensitize SW620 cells to anoikis, which is not what we observed.

In conclusion, whether or not they undergo anoikis, suspended colon cancer cells are sensitive to the BH3mimetic ABT-737. This indicates that cancer cells that have detached from the extracellular matrix might go through a transient window where they can be killed by compounds such as Navitoclax or ABT-199, which would give anti-metastatic properties to these agents.

\section{Materials and Methods}

Cell lines and reagents. SW480 and SW620 human colon carcinoma cell lines were obtained from the American Type Culture Collection (ATCC). They were routinely maintained in Dulbecco's minimal essential medium (DMEM) (Gibco, Saint Aubin, France) supplemented with 10\% heat-inactivated fetal bovine serum, $1 \%$ sodium pyruvate, $0.1 \mathrm{mg} / \mathrm{ml}$ streptomycin, $100 \mathrm{U} / \mathrm{ml}$ penicillin at $37^{\circ} \mathrm{C}$ in a $5 \% \mathrm{CO}_{2}$-humidified atmosphere. For the cultures in suspension, 2 units $/ \mathrm{ml}$ of dispase (Roche, Meylan, France) was added to the culture medium to obtain single cell suspensions. Dispase is a bacterial neutral protease with mild proteolytic activity, which is mainly used for the isolation and routine passaging of primary cells. It can also detach adherent cells from the culture vesse ${ }^{49}$ by digesting the extracellular matrix ${ }^{50}$ and dissociating cellular aggregates. ${ }^{51}$ Dispase is not inactivated by serum and, given its lack of toxicity, is a very efficient mean for keeping adherent cells in unicellular suspension.

TRAIL sensitivity of SW480 clones was tested by culturing the cells in the presence of Flag-tagged recombinant TRAIL $(25 \mathrm{ng} / \mathrm{ml})$ (Alexis Biochemicals, Villeurbanne, France) and anti-Flag (M2) antibody (Sigma Aldrich, Lyon, France) for various times.

Stock solutions of Q-VD-OPH (Biovision, Nanterre, France), Ly294002 (Calbiochem, Darmstadt, Germany) and PD0325901 (Sigma Aldrich) were prepared in DMSO at $10 \mathrm{mM}$ and used at $10 \mu \mathrm{M}$ final concentration. ABT-737 (Selleckchem, Souffelweyersheim, France) was also prepared in DMSO at $10 \mathrm{mM}$ and used at $1 \mu \mathrm{M}$ final concentration.

The following antibodies were used: anti-Bax (N-20, Santa Cruz Biotechnology, Inc, Heildelberg, Germany), anti-Bid (R\&D Systems, Lille, France), anti- cleaved caspase-3 (Cell Signaling, Saint Quentin en Yvelines, France), anti-Hsc70 (B-6, Santa Cruz Biotechnology, Inc), anti-Mcl-1 (S-19, Santa Cruz Biotechnology, Inc), antiBcl-2 (H-100, Santa Cruz Biotechnology, Inc), anti-Bcl-xL (BD Transduction Laboratories, Le Pont de Claix, France), anti-Bim (Cell Signaling), anti-pErk (Cell Signaling), anti-Erk total (Cell Signaling), anti-actin (MBL, Nanterre, France) and antiFADD (BD Transduction Laboratories).

Flow cytometry analysis of apoptotic cells. Suspended cells maintained in culture from $24-72 \mathrm{~h}$ were washed in PBS (phosphate-buffered saline), fixed in $70 \%$ cold ethanol and stored at $-20{ }^{\circ} \mathrm{C}$ for at least $12 \mathrm{~h}$. After two washes in PBS, cells were resuspended and stained in $0.5 \mathrm{ml}$ of PBS containing $50 \mu \mathrm{g} / \mathrm{ml}$ propidium iodide. The fluorescence intensity of propidium iodide was assessed with a FACSCalibur flow cytometer (Becton Dickinson, Le Pont de Claix, France) and analyzed using the CellQuest (Becton Dickinson) software. The percentage of cells with apoptotic nuclei, distinguished by their hypodiploid DNA content (sub-G0/G1 peak), ${ }^{52}$ was assessed for each histogram. 
Western blotting and immunoprecipitation. Cells were lysed with icecold NP40 lysis buffer $(30 \mathrm{mM}$ Tris pH 7.5, $150 \mathrm{mM} \mathrm{NaCl}, 10 \mathrm{mM} \mathrm{NaF}, 1 \mathrm{mM}$ EDTA, $1 \mathrm{mM}$ EGTA, 10\% Glycerol, $10 \mathrm{mM} \mathrm{NaPP}$ and 1\% NP40) containing $1 \mathrm{mM}$ phenylmethylsulfonylfluoride, $1 \mathrm{mM}$ sodium orthovanadate $\left(\mathrm{Na}_{3} \mathrm{VO}_{4}\right)$ and $1 / 100$ protease cocktail inhibitors (Sigma, Lyon, France). All lysates were clarified by centrifugation at $15000 \times \mathrm{g}$ for $15 \mathrm{~min}$ at $4{ }^{\circ} \mathrm{C}$. Protein concentrations were assessed using the Bradford assay (BioRad, Hercules, CA, USA). Proteins amounting to $50 \mu \mathrm{g}$ were prepared in loading Laemmli's buffer $(60 \mathrm{mM}$ Tris-HCL $\mathrm{pH} 6.8,0.18 \mathrm{M} \beta$-mercaptoethanol, $2 \%$ SDS, $10 \%$ glycerol and $0.005 \%$ bromophénol blue), boiled for $5 \mathrm{~min}$, subjected to sodium dodecyl sulfatepolyacrylamide gel electrophoresis and transferred on ice for $1.5 \mathrm{~h}$ to polyvinylidenedifluoride membrane (Amersham Hybond-LFP, GE healthcare, Velizy-Villacoublay, France). After blocking for at least $1 \mathrm{~h}$ in Tris-buffered saline supplemented with $5 \%$ BSA and $0.5 \%$ Tween-20 (TBS-T), membranes were probed with the appropriate primary antibodies in TBS-T overnight. Proteins were visualized by using Lycor secondary antibodies and Odyssey detection material.

For immunoprecipitation experiments, cells were lysed with CHAPS lysis buffer ( $30 \mathrm{mM}$ Tris pH 7.5, $150 \mathrm{mM} \mathrm{NaCl}, 10 \mathrm{mM} \mathrm{NaF}, 1 \mathrm{mM}$ EDTA, $1 \mathrm{mM}$ EGTA, $10 \%$ Glycerol, $10 \mathrm{mM} \mathrm{NaPP}$ and $1 \%$ CHAPS) containing $1 \mathrm{mM}$ phenylmethylsulfonylfluoride, $1 \mathrm{mMsodium}$ orthovanadate $\left(\mathrm{Na}_{3} \mathrm{VO}_{4}\right)$ and $1 / 100$ protease cocktail inhibitors). Nuclear pellets and debris were removed by centrifugation, at $15000 \times g$ for $15 \mathrm{mn}$ at $4{ }^{\circ} \mathrm{C}$. Six milligrams of proteins from cell lysates were incubated for $1 \mathrm{~h}$ at $4{ }^{\circ} \mathrm{C}$ with the anti-Bim antibodies. Protein-G beads were added to the immune complexes for $45 \mathrm{~min}$ and washed five times with ice-cold CHAPS lysis buffer. Purified immunoprecipitates, immobilized on protein-G beads, were mixed with an equal volume of Laemmli's buffer $2 x$, boiled for $5 \mathrm{~min}$ and further analyzed by means of western blot for both Bim and Bcl-2 content.

Small-interfering RNA-mediated silencing of Bim. In $3 \mathrm{ml}$ of culture medium, $3 \times 10^{5}$ cells were transfected with Bim siRNA or irrelevant siRNA (Ambion Life Technologies, Saint Aubin, France). Each siRNA was used at $20 \mathrm{nM}$ final concentration. INTERFERin $(20 \mu$ l, Polyplus transfection, Ozyme, Saint Quentin en Yvelines, France) was incubated with siRNA duplex in $800 \mu$ l of DMEM without serum for $20 \mathrm{~min}$ at room temperature. The mixture was then added to the cells, which were transferred to culture plates and incubated at $37^{\circ} \mathrm{C}$. Seventytwo hours after transfection, cells were detached with culture medium containing 2 units/ml of dispase, cultured in this medium for 24,48 or $72 \mathrm{~h}$ and the percentage of apoptotic cells was quantified as described above. Extinction of Bim expression by the Bim siRNA was monitored by means of western blot throughout the culture in suspension.

Stable transfection of FADD.dn in SW480 cells. The pcDNA3/ FADD.dn vector encodes for a truncated form of FADD protein deleted of its two DED domains and thus unable to recruit caspase-8. SW480 cells were transfected $5 \mu \mathrm{g}$ of either pcDNA3/FADD.dn or pcDNA3 empty vector with the use of JetPei (Polyplus transfection). Transfected cells were selected with neomycin $(400 \mu \mathrm{g} / \mathrm{ml})$ and then cloned.

Cell fractionation. We used the 'cell fractionation kit' (catalog no. 9038) from Cell Signaling Technology according to manufacturer's instructions. This methodology is detergent-based ${ }^{53}$ and is performed on ice. Cell pellet is resuspended in a first, digitonin-based, buffer for $5 \mathrm{mn}$ followed by a centrifugation at $500 \times g$. The supernatant is the cytosolic protein-enriched fraction. The pellet is resuspended in a second, triton-based buffer for $5 \mathrm{mn}$ and centrifuged at $8000 \times g$. The supernatant is the membrane and organellar protein-enriched fraction, which contains, among others, mitochondria-associated proteins. The remaining pellet, which we did not use, contains the actin cytoskeleton and the nuclear proteins. Given that microtubules depolymerize within minutes on ice, tubulin and all associated proteins, including dynein motor complex-bound Bim for our purpose, end up in the cytosolic fraction.

\section{Conflict of Interest}

The authors declare no conflict of interest.

Acknowledgements. We thank Philippe Mauduit, Frank Gesbert and Eric Rubinstein for fruitful comments and critical reading of the manuscript. AM-A is supported by a grant from the Ministère de la Recherche et de l'Enseignement Supérieur and by NRB. JB is supported by ARC and INSERM.

1. Frisch S, Francis H. Disruption of epithelial cell-matrix interactions induces apoptosis. J Cell Biol 1994; 124: 619-626.

2. Danial NN, Korsmeyer SJ. Cell death: critical control points. Cell 2004; 116: 205-219.

3. Gilmore AP. Anoikis. Cell Death Differ 2005; 12: 1473-1477.

4. Chambers AF, Groom AC, MacDonald IC. Dissemination and growth of cancer cells in metastatic sites. Nat Rev Cancer 2002; 2: 563-572.

5. Ruoslahi E, Reed JC. Anchorage dependence, integrins, and apoptosis. Cell 1994; 77 : 477-478.

6. Grossmann J. Molecular mechanisms of 'detachment-induced apoptosis-Anoikis'. Apoptosis 2002; 7: 247-260.

7. Cory S, Adams JM. The Bcl2 family: regulators of the cellular life-or-death switch. Nat Rev Cancer 2002; 2: 647-656.

8. Martinou JC, Youle RJ. Mitochondria in apoptosis: Bcl-2 family members and mitochondrial dynamics. Dev Cell 2001; 21: 92-101.

9. Letai A, Bassik MC, Walensky LD, Sorcinelli MD, Weiler S, Korsmeyer SJ. Distinct BH3 domains either sensitize or activate mitochondrial apoptosis, serving as prototype cancer therapeutics. Cancer Cell 2002; 2: 183-192.

10. Cartron PF, Gallenne T, Bougras G, Gautier F, Mareno F, Vusio P et al. The first alpha helix of Bax plays a necessary role in its ligand-induced activation by the $\mathrm{BH}$-only proteins Bid and PUMA. Mol Cell 2004; 16: 807-818.

11. Oltersdorf T, Elmore SW, Shoemaker AR, Armstrong RC, Augeri DJ, Belli BA et al. An inhibitor of Bcl-2 family proteins induces regression of solid tumours. Nature 2005; 435 : 677-681.

12. Richardson A, Kaye SB. Pharmacological inhibition of the Bcl-2 family of apoptosis regulators as cancer therapy. Curr Mol Pharmacol 2008; 1: 244-254.

13. Kutuk $O$, Letai A. Alteration of the mitochondrial apoptotic pathway is key to acquired paclitaxel resistance and can be reversed by ABT-737. Cancer Res 2008; 68: 7985-7994.

14. Kim KW, Moretti L, Mitchell LR, Jung DK, Lu B. Combined Bcl-2/mammalian target of rapamycin inhibition leads to enhanced radiosensitization via induction of apoptosis and autophagy in non-small cell lung tumor xenograft model. Clin Cancer Res 2009; 15: 6096-6105.

15. Corcoran RB, Cheng KA, Hata AN, Faber AC, Ebi H, Coffee EM et al. Synthetic lethal interaction of combined BCL-XL and MEK inhibition promotes tumor regressions in KRAS mutant cancer models. Cancer Cell 2013; 23: 121-128.

16. Rahmani M, Mayo Aust M, Attkisson E, Williams DC Jr, Ferreira-Gonzalez A, Grant S. Dual inhibition of $\mathrm{Bcl}-2$ and $\mathrm{Bcl}-\mathrm{xL}$ strikingly enhances PI3K inhibition-induced apoptosis in human myeloid leukemia cells through a GSK-3 and Bim-dependent mechanism. Cancer Res 2013; 73: 1340-1351.

17. Souers AJ, Leverson JD, Boghaert ER, Ackler SL, Catron ND, Chen J et al. ABT-199, a potent and selective $\mathrm{BCL}-2$ inhibitor, achieves antitumor activity while sparing platelets. Nat Med 2013; 19: 202-208.

18. Certo M, Del Gaizo Moore V, Nishino M, Wei G, Korsmeyer S, Armstrong SA et al. Mitochondria primed by death signals determine cellular addiction to antiapoptotic BCL-2 family members. Cancer Cell 2006; 9: 351-365.

19. Del Gaizo Moore V, Brown JR, Certo M, Love TM, Novina CD, Letai A. Chronic lymphocytic leukemia requires $\mathrm{Bcl} 2$ to sequester prodeath Bim explaining sensitivity to $\mathrm{Bcl} 2$ antagonist ABT-737. J Clin Invest 2007; 117: 112-121.

20. Reginato MJ, Mills KR, Paulus JK, Lynch DK, Sgroi DC, Debnath $\mathrm{J}$ et al. Integrins and EGFR coordinately regulate the pro-apoptotic protein Bim to prevent anoikis. Nat Cell Biol 2003; 5: 733-740.

21. Hausmann M, Leucht $\mathrm{K}$, Ploner $\mathrm{C}$, Kiessling S, Villunger A, Becker $\mathrm{H}$ et al. BCL-2 modifying factor (BMF) is a central regulator of anoikis in human intestinal epithelial cells. J Biol Chem 2011; 286: 26533-26540.

22. Leibovitz A, Stinson JC, McCombs WB 3rd, McCoy CE, Mazur KC, Mabry ND. Classification of human colorectal adenocarcinoma cell lines. Cancer Res 1976; 36: 4562-4569.

23. Wang $\mathrm{P}$, Valentijn AJ, Gilmore AP, Streuli $\mathrm{CH}$. Early events in the anoikis program occur in the absence of caspase activation. J Biol Chem 2003; 278: 19917-19925.

24. Frisch SM. Evidence for a function of death-receptor-related, death-domain-containing proteins in anoikis. Curr Biol 1999; 9: 1047-1049.

25. Laguinge LM, Samara RN, Wang W, El-Deiry WS, Corner G, Augenlicht L et al. DR5 receptor mediates anoikis in human colorectal carcinoma cell lines. Cancer Res 2008; 68: 909-917.

26. Ndozangue-Touriguine $\mathrm{O}$, Sebbagh $\mathrm{M}$, Merino $\mathrm{D}$, Micheau $\mathrm{O}$, Bertoglio J, Bréard J. A mitochondrial block and expression of XIAP lead to resistance to TRAIL-induced apoptosis during progression to metastasis of a colon carcinoma. Oncogene 2008; 27: 6012-6022.

27. Tiwari N, Gheldof A, Tatari M, Christofori G. EMT as the ultimate survival mechanism of cancer cells. Sem Cancer Biol 2012; 22: 194-207.

28. Hazan SB, Qiao R, Keren R, Badano I, Suyama K. Cadherin switch in tumor progression Ann N Y Acad Sci 2004; 1014: 155-163. 
29. Kumar S, Park SH, Cieply B. A pathway for the control of anoikis sensitivity by E-cadherin and epithelial-to-mesenchymal transition. Mol Cell Biol 2011; 31: 4036-4051.

30. Li G, Satyamoorthy K, Herlyn M. N-cadherin-mediated intercellular interactions promote survival and migration of melanoma cells. Cancer Res 2001; 61: 3819-3825.

31. Hewitt RE, McMarlin A, Kleiner D, Wersto R, Martin P, Tsokos M et al. Validation of a model of colon carcinoma progression. J Pathol 2000; 192: 446-454.

32. Woods NT, Yamaguchi H, Lee FY, Bhalla KN, Wang Wang HG. Anoikis initiated by Mcl-1 degradation and Bim induction, is deregulated during oncogenesis. Cancer Res 2007; 67: 10744-10752.

33. Nijhawan D, Fang M, Traer E, Zhong Q, Gao W, Du F et al. Elimination of Mcl-1 is required for the initiation of apoptosis following ultraviolet irradiation. Genes Dev 2003; 17: $1475-1486$.

34. O'Connor L, Strasser A, O'Reilly LA, Hausmann G, Adams JM, Cory S et al. Bim: a novel member of the Bcl-2 family that promotes apoptosis. EMBO J 1998; 17: 384-395.

35. Puthalakath H, Villunger A, O'Reilly LA, Beaumont JG, Coultas L, Cheney RE et al. Bmf: a proapoptotic $\mathrm{BH}$ 3-only protein regulated by interaction with the myosin $\mathrm{V}$ actin motor complex, activated by anoikis. Science 2001; 293: 1829-1832.

36. Schmelzle T, Mailleux AA, Overholtzer M, Carroll JS, Solimini NL, Lightcap ES et at. Functional role and oncogene-regulated expression of the BH3-only factor Bmf in mammary epithelial anoikis and morphogenesis. Proc Natl Acad Sci USA 2007; 104: 3787-3792.

37. Puthalakath H, Huang DCS, O'Reilly LA, King SM, Strasser A. The proapoptotic activity of the $\mathrm{Bcl}-2$ member Bim is regulated by interaction with the dynein motor complex. Molecular Cell 1999; 3: 287-296.

38. Akiyama T, Dass CR, Choong PFM. Bim-targeted cancer therapy: a link between drug action and underlying molecular changes. Mol Cancer Ther 2009; 8: 3173-3180.

39. Chen J, Jin S, Abraham V, Huang X, Liu B, Mitten MJ et al. The Bcl-2/Bcl-XL/Bcl-w inhibitor, Navitoclax, enhances the activity of chemotherapeutic agents in vitro and in vivo. Mol Cancer Ther 2011; 10: 2340-2349.

40. Bertin-Cifti J, Bérré B, Le Pen J, Maillet L, Couriaud C, Juin P et al. pRb/E2F-1-mediated caspase-dependent induction of Noxa amplifies the apoptotic effects of the $\mathrm{Bcl}-2 / \mathrm{Bcl}-\mathrm{xL}$ inhibitor ABT-737. Cell Death Diff 2013; 20: 755-764.

41. Deng J, Carlson N, Takeyama K, Dal Cin P, Shipp M et al. BH3 profiling identifies three distinct classes of apoptotic blocks to predict response to ABT-737 and conventional chemotherapeutic agents. Cancer Cell 2007; 12: 171-185.

42. Letai A. Restoring cancer's death sentence. Cancer Cell 2006; 10: 343-345.
43. Kim YN, Koo KH, Sung JY, Yun UJ, Kim H. Anoikis resistance: an essential prerequisite for tumor metastasis. Int J Cell Biol 2012; 2012: 306879.

44. Brabletz T. To differentiate or not- routes toward metastasis. Nature Rev Cancer 2012; 12 425-436.

45. Konopleva M, Contractor R, Tsao T, Samudio I, Ruvolo PP, Kitada S et al. Mechanisms of apoptosis sensitivity and resistance to the BH3-mimetic ABT-737 in acute myeloid leukemia. Cancer Cell 2006; 10: 375-388.

46. Van Delft MF, Wei AH, Mason KD, Vandenberg CJ, Chen L, Czabotar PE et al. The BH3-mimetic ABT-737 targets selective $\mathrm{Bcl}-2$ proteins and efficiently induces apoptosis via Bak/Bax if Mcl-1 is neutralized. Cancer Cell 2006; 10: 389-399.

47. Luciano F, Jacquel A, Colosetti P, Herrant M, Cagnol S, Pages G et al. Phosphorylation of Bim-EL by Erk $1 / 2$ on serine 69 promotes its degradation via the proteasome pathway and regulates its proapoptotic function. Oncogene 2003; 22: 6785-6793.

48. Maurer U, Charvet C, Wagman AS, Dejardin E, Green DR. Glycogen synthase kinase-3 regulates mitochondrial outer membrane permeabilization and apoptosis by destabilization of MCL-1. Mol Cell 2006; 21: 749-760.

49. Cassiman JJ, Brugmans M, Van den Berghe H. Growth and surface properties of dispase dissociated human fibroblasts. Cell Biol Int Rep 1981; 5: 125-132.

50. Stenn KS, Link R, Moellmann G, Madri J, Kuklinska E. Dispase a neural protease from Bacillus polymyxa, is a powerful fibronectinase and type IV collagenase. J Invest Dermatol 1989; 93: 287-290.

51. Craviso GL. Generation of functionally competent single bovine adrenal chromaffin cells from cell aggregates using the neutral protease dispase. J Neurosci Methods 2004; 137: 275-281.

52. Nicoletti I, Migliorati G, Pagliacci M.C, Grignani F, Riccardi C. A rapid and simple method for measuring thymocyte apoptosis by propidium iodide staining and flow cytometry. J Immunol Methods 1991; 139: 271-279.

53. Michelsen U, von Hagen J. Isolation of subcellular organelles and structures. Methods Enzymol 2009; 463: 305-328.

(c) (i) (2) Cell Death and Disease is an open-access journal By licensed under a Creative Commons Attribution-NonCommercialShareAlike 3.0 Unported License. To view a copy of this license, visit http://creativecommons.org/licenses/by-nc-sa/3.0/ 